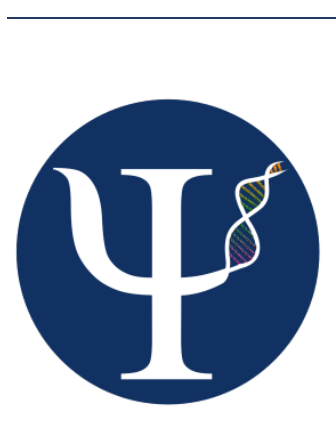

\title{
"Kioscos Vive Digital": una estrategia digital y de conectividad para promover la inclusión social en comunidades rurales en Colombia. "Kioscos Vive Digital": A digital strategy to promote the connectivity and social inclusion in rural communities in Colombia
}

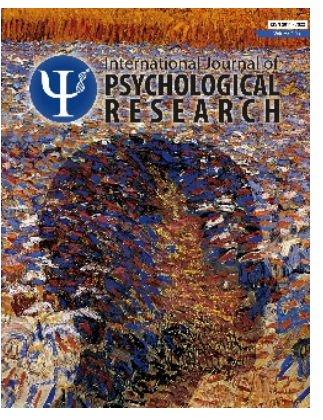

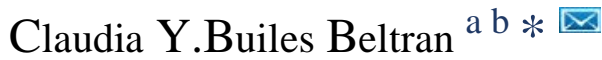

R e f l e x i ó n

${ }^{a}$ Directora General Gemprecol, Colombia.

${ }^{b}$ Investigadora Asociada, Grupo de Investigación Salud Comportamenal y Organizacional, Universidad de San Buenaventura, Medellín, Colombia

\section{ARTICLE INFO}

\section{RESUMEN}

Este artículo explora los alcances de las Tecnologías de la Información y la Comunicación (TIC) en la recuperación del tejido social y el desarrollo de comunidades rurales apartadas, de veredas y corregimientos, con poco acceso a recursos informáticos y de conectividad en Colombia. Un modelo que ha sido implementado exitosamente en Suramérica, y que contribuye al desarrollo de procesos de inclusión social a partir del acceso a las comunidades digitales, a la educación virtual y a las redes sociales como mecanismos de conectividad, que favorecen referentes identitarios y que proveen el desarrollo social con equidad. Para esto, los "Kioscos Vive Digital", se constituyen en una estrategia de acceso a la conectividad en función de la recuperación de las comunidades a través de la interacción en un escenario virtual probado propositivamente por la Psicologia social y las neurociencias, y que beneficia la consolidación de redes sociales y la entrada a la información como eje central para el equilibrio y la inclusión social.

\section{ABSTRACT}

This article explores the scope of CIT in the recovery of the social background and the development of rural and isolate communities, with little access to computing resources and internet in Colombia. This model has been implemented successfully in South America, contributing to the development of processes of social inclusion for access to digital communities, virtual education and social networks, as mechanisms of connectivity which favor concerning a social identity and provide social development with equity. For this, the "kiosk lives Digital", constitute a strategy for access to connectivity based on the recovery of communities through interaction in a virtual scenario tested purposefully by social psychology and neuroscience which benefit the consolidation of social networks and the entrance to the information as a central axis for balance and social inclusion.
Article history:

Received: 22-08-2015

Revised: 01-10-2015

Accepted: 01-12-2015

Palabras calve: kioscos vive digital, inclusión social, TIC, psicología social, comunicación digital.

Key words: kioscos vive digital, social inclusion, CIT, social psychology, digital communications

\footnotetext{
* Corresponding author: Claudia Y Builes Beltran, Gemprecol, Colombia. Email address: coordinaciondecampo@genmprecol.com.co
} 


\section{INTRODUCCIÓN}

Con la implementación del World Wide Web (WWW), la red informática del mundo se hizo posible la distribución de documentos en hipertexto como recurso abierto vía internet; esto permitió a los usuarios acceder a contenidos web que presentaban texto, videos e imágenes y contenidos multimedia entre otros. Para finales de 1990 el flujo de información contribuyó a esclarecer cómo funcionaban las economías, las organizaciones, las dinámicas culturales y los movimientos sociales entre otros; así, cuando el uso de Internet se generalizó en la sociedad occidental, trajo como consecuencia la conversión paulatina y exponencial de nuestro acervo personal e interacción social a un código binario, con todo esto, la posibilidad que las nuevas tecnologías habilitaron en cuanto a la velocidad de flujos de información y dinámicas sociales, ha producido cambios radicales en la forma de entender la economía, las organizaciones y el trabajo, pero también la cultura, la política y los movimientos sociales.

Este artículo, delinea los alcances que han tenido las TIC (Tecnologías de la Información y la Comunicación), a través de estrategias como los Kioscos Digitales, centros que usan plataformas de conectividad digital para promover el cambio en la comunidad desde diferentes alternativas de inclusión social en el contexto cultural desde lo que implican los procesos de conectividad digital; así mismo, delinean desde el reconocimiento del capital humano y los aprendizajes colaborativos en lugares rurales de difícil acceso tecnológico, pedagógico y con fallas en el acceso a servicios digitales, la comunicación eficiente para la promoción de hábitos y estilos de vida saludables.

\section{TIC Y VÍNCULO SOCIAL}

Para este contexto, las Tecnologías de la Información y la Comunicación, (TIC), se definen como el conjunto de herramientas informáticas requeridas para gestionar la información, no obstante, más allá de la adquisición, el registro, el almacenamiento y la recuperación de datos, ellas proporcionan una miríada de soluciones con impacto directo en el tejido social, ya que los entornos multimedia y en línea se ajustan de manera versátil, dinámica e interactiva a las necesidades de la comunidad en pro de mediatizar el conocimiento en un escenario de globalización de la información. Por tanto, las TIC constituyen un referente actual en la
Psicologia social construccionista ya que habilita nuevas formas de construcción de identidad a partir de los nuevos mecanismos de inclusión que se avalan a través de las nuevas tecnologías de comunicación digital.

Un ejemplo del impacto de la conectividad es el proporcionado por las redes sociales, que en el concepto de Kaplan y Haenlein, se constituyen en un "grupo de aplicaciones basadas en Internet desarrolladas a partir de la Web 2.0, y que permiten la creación y el intercambio de contenidos generados por el usuario" (Kaplan, A. M, 2015); de hecho las redes sociales permean nuestras sociedades $y$ nuestra cultura, una prueba de ello son los 1.5 billones de usuarios regulares en el mundo para la red social Facebook, esto es, uno de cada cinco personas en el planeta tiene un perfil en esta red social (Bercovici, 2010), para 2014 la población de Estados Unidos era de 318.9 millones de personas, de las cuales 193 millones eran usuarios activos en Facebook y para América del Sur se habían documentado a 2015 la cifra aproximada de 299. Millones de personas con cuenta en Facebook, que para el caso de Colombia con una población cercana a los 48 millones de personas, 24 millones se sabe que tienen un perfil abierto en Facebook (https://www.owloo.com/). Es justamente por este marco de conectividad digital que los investigadores de diferentes disciplinas como la psicología, la política, la sociología, el consumo y el marketing entre otras, se han enfocado en profundizar sobre los fenómenos sociales y culturales que emergen en el mundo de las redes sociales y la comunicación.

Así mismo, es indudable lo que se ha logrado en el mapa digital de las conexiones; el desarrollo científico y tecnológico de los últimos 40 años en el mundo, se ha beneficiado del intercambio digital, un ejemplo de ello es lo alcanzado por países como Japón, Noruega y Suecia cuyos altos índices de conectividad han promovido mejores resultados educativos para la población (Christakis \& Fowler 2009).

Quizás una de las dificultades más frecuentes en contextos de cambio cultural relacionado a los procesos digitales, tiene que ver con la "tecnofobia" o rechazo a las tecnologías por el imaginario negativo y la perdida potencial de vínculos con la realidad, como también, el temor a la adicción con la virtualidad de las relaciones que van perdiendo naturalidad y se consideran artificiales. Sin embargo estos argumentos son controversiales, pues es relevante mencionar que el escenario digital es un mediador y modulador de las identidades individuales y colectivas para los vínculos sociales; de hecho, el acceso a lo digital es un eje de 
International Journal of Psychological Research

diferenciación evolutivo en la postmodernidad que dinamiza el conocimiento y favorece la adaptación de las personas en los diferentes medios para los cuales debe exhibir habilidades de actualización, cambio y resiliencia.

\section{NEUROCIENCIAS Y TIC}

Son varios los argumentos a favor de como la evolución de procesos cognitivos vinculados a lo que conocemos como "cerebro digital", constituyen ventajas en el alcance de la conexión digital. Al respecto, los experimentos realizados por el Dr. Small y colaboradores han perimtido compar la actividad cerebral de "nativos digitales" (usuarios con aprendizajes previos y con uso frecuente de computadores), con personas "inmigrantes digitales" (personas con menos experiencia en entornos virtuales y acceso a computadores), cuando ambos grupos realizan búsquedas por Google y navegaban en la red, este estudio determinó que el funcionamiento ejecutivo relacionado con la planificación y que se asocian a nivel cerebral con el córtex prefrontal dorsolateral se desarrollaban con más destreza en los "nativos digitales", sin embargo después de cinco días de entrenamiento, el cerebro de los "inmigrantes digitales" presentaban niveles similares de activación cerebral que los "nativos digitales" (Small \& Vorgan, 2008). Adicionalmente y en contradicción a los mitos urbanos propios de la tecnofobia, Steven Pinker resaltó que el cerebro en red no se remodela como un amasijo de barro, si bien el aprendizaje y el alcance digital redirecciona nuestras experiencias al permitirnos nuevos referentes de análisis, los dispositivos básicos asociados a nuestro procesamiento de la información no se ven alterados (Pinker, 2009).

En lo relacionado a como los sistemas neurales se relacionan con competencias involucradas a los escenarios virtuales de conectividad con la red social, Meshi y colaboradores han sugerido tres dominios fundamentales; la cognición social o la capacidad de procesar e interpretar las situaciones sociales principalmente la mentalización que da cuenta de la intencionalidad social y las emociones vinculadas con la comunicación y los procesos del Yo, los cuales favorecen la autorreflexión, el automonitoreo y el autoreconocimiento, y los sistemas de recompensa social, los cuales estan estrechamente relacionados con la capacidad de generar adherencias sociales que cada uno percibe como benéficas (reputación social en la red social). Estos tres constructos estrechamente relacionados a la Neurociencia Social

\section{"Kioscos Vive Digital": una estrategia digital}

han sido profundamente estudiados, y a través de técnicas de neuroimagen se destacan correlatos cerebrales asociados con cada uno de estos componentes: para los procesos de mentalización la activación de áreas de la corteza prefrontal dorsomedial, para los procesos del Yo la corteza posterior cingulada y la corteza prefrontal medial, y para los procesos de recompensa la corteza prefrontal ventromedial y el área ventral tegmental. Con estos hallazgos se soporta cada vez más las implicaciones que tienen los procesos cognitivos en la dinámica digital para los procesos de interacción social (Meshi, et al. 2015).

De otro lado, el acceso a las TIC escalan un llamado fundamental que como especie tenemos a cooperar como raíz consustancial para entender nuestra naturaleza social; la conexión virtual y las redes sociales son abundantes en los mecanismos de cooperación, uno especialmente importante en la conectividad virtual es aquel donde un usuario "A" coopera con otro "B"(desconocido), basándose en la reputación que "B" tiene debido a su comportamiento con otros, de tal forma que, haber cooperado con otros aumenta su reputación y por tanto, la probabilidad de que reciba cooperación de otros, este mecanismo es de especial interés pues refiere directamente a lo que sucede en escenarios de conectividad virtual, en donde los individuos cooperan frecuentemente a través de chats, foros, blogs, imágenes, videos y recursos web, aun cuando la cooperación no necesariamente sea recíproca, pues consolida en el imaginario virtual la percepción de beneficios futuros por la reputación que gana el cooperante de haber cooperado en el pasado, una respuesta que solo se ha documentado en los humanos (Roberts, 2015).

\section{4. "KIOSCOS VIVE DIGITAL Y TEJIDO SOCIAL"}

En Colombia como en los países latinoamericanos que se estructuran como multiculturales, plurietnicos, diversos y biodiversos, los procesos identitarios son relevantes para la construcción de comunidad de cara al desarrollo del tejido social. En este sentido el acceso a recursos que provean comunicación y formación eficientes en tiempo y con profundidad, favorecen la transformación de la realidad social y política, el fomento a los valores, la cooperación, la equidad y la inclusión social en un contexto político de responsabilidad social.

En este marco referencial, con el advenimiento de políticas estatales del Ministerio de Tecnologías de la Información y las Comunicaciones 
International Journal of Psychological Research

(MinTIC), en Colombia (Comunicaciones. 2016), y a través de propuestas como "Kioscos Vive Digital", cuyo propósito central es facilitar el acceso para que la poblaciones de las zonas rurales (veredas y corregimientos) o de difícil acceso del país tengan acceso a las TIC, a través de plataformas que se configuran con un diseño e instalación eficientes para el desarrollo de competencias digitales, se privilegia el desarrollo de las comunidades en pro de la inserción social superando la brecha digital y de información. Los entornos virtuales se articulan desde dos vías de desarrollo, la primera es las relaciones con el otro en el crecimiento de dinámicas de comunicación online y en red, relacionando intereses comunes y reconocimiento de la diferencia como pilar central de la construcción de identidad y tejido social; la segunda alternativa es la educación como disciplina de crecimiento personal y profesional que contribuye a la autonomía y a la construcción de roles adaptativos dentro de la comunidad como soporte central del desarrollo individual y construcción de talento como diferenciador en la red social.

"Los Kioscos Vive Digital (KVD)", permiten entonces, implementar en el paradigma contemporáneo de la comunicación e información virtual, la posibilidad de reflexionar en torno a lo subjetivo e identitario para consolidar comunidades cooperantes y de autogestión de procesos que transforman tejido social. Por tanto, esta alternativa de relación digital puede direccionar el cambio social como núcleo de los movimientos sociales que promueven la equidad, el acceso a la información, la necesidad de reconocimiento, la organización, el respeto a la diferencia y el fomento a la innovación desde el capital humano en una sociedad que debe resaltar el bien común.

De otro lado el acceso a las TIC que proveen los KVD, contribuyen a generar políticas de inclusión social, en tanto que pueden funcionar como herramienta de reconocimiento de necesidades comunitarias, a través de la conectividad el usuario tiene la posibilidad de clasificar sus prioridades dentro de su grupo social, étnico y cultural, lo cual le permitiría a los líderes organizacionales de turno establecer de acuerdo a la clase social, la edad, los niveles de escolaridad, el trabajo, el tipo de comunidad, el oficio, el uso y el propósito de la conexión virtual y los intereses de formación que tienen las personas, la posibilidad de, dinamizar los cambios sociales a través de políticas relevantes en pro de las comunidades.

Los KVD son congruentes con las dinámicas de globalización ya que comunicación virtual y el acceso a la información no están limitadas por la
"Kioscos Vive Digital": una estrategia digital

dimensión geográfica, provee niveles de comunicación temporal sincrónica o asincrónica y ofrece a las personas la autonomía suficiente para la construcción y el alcance de sus propias redes sociales.

A la vez el acceso a través de estas propuestas digitales en Colombia plantean varios retos para el desarrollo de la construcción de tejido social y comunidades saludables en la dinámica de entornos virtuales. Inicialmente, cambiar en el imaginario colectivo de usuarios potenciales, las ideas y conceptos que sugieren los efectos negativos del uso del ordenador en la virtualidad, esto relacionado a que la comunicación digital y los vínculos sociales en red promueven las relaciones frágiles e impersonales entre las personas, generándose dificultades en llegar a consensos de grupo al interior de las comunidades virtuales, y por la defensa frente a la autonomía de la interacción entre los usuarios, puede incrementarse la conducta habitual de comportamientos hostiles y poco prosociales en las relaciones que se crean; adicionalmente, el incremento de información errónea y falsa, aspecto común en las redes sociales, terminaria vulnerando el propósito de los entornos virtuales saludables.

En buena parte la propuesta potencial de los KVD como estrategia de cambio social a partir de entornos virtuales saludables, está en concordancia con lo formulado por Nancy K. Baym, en lo que significa incorporarse a redes sociales virtuales que promueven la cooperación y el enlace por intereses comunes para la vida cotidiana de las personas (Baym, 2010); así mismo, es indudable que la comunicación de contenidos sociales y emocionales a través de lo digital pueden ser similares o incluso superiores a los que pueden darse cara a cara (Reid, 1991); además, el equilibrio de estatus entre las personas y el incremento del círculo social con las alternativas potenciales de incorporar las redes personales virtuales al espacio real potencial, proveen el desarrollo de la empatía cognitiva por la presencia de intereses comunes, creando un nicho social distintivo y autorregulado por cada uno de los usuarios.

Finalmente, las repercusiones sociales que conlleva la estrategia de los KVD son relevantes para el desarrollo de las comunidades que tienen poco o nulo acceso a recursos digitales, como también, para aquellas que adolecen de la falta de procesos de aprendizaje suficientes para vincularse a las dinámicas de virtualidad. Varios de los componentes centrales que soportan los KVD como mecanismos de inclusión y capacitación social son: 
- El desarrollo de las habilidades personales para inmigrantes digitales, suficientes para acceder adecuadamente a los procesos de aprendizaje y conectividad social en escenarios digitales.

- El reconocimiento de las personas que acceden a los KVD de los valores y el contexto social que definen su comunidad como recurso fundamental de acervo social local y regional

- El acceso a entretenimiento y al uso del tiempo libre y formativo en pro de la diferenciación cultural de roles de género y de inclusión

- La promoción a través de la conectividad digital del uso de recursos mediáticos como puerta de entrada al universo simbólico, ofreciendo a las personas múltiples alternativas para la configuración de identidades y promoviendo estilos de vida saludables desde un contexto digital ventajoso

- Facilitar formas de relación al interior de la comunidad o de trabajos colaborativos como proceso social de construcción del conocimiento, para robustecer las redes de innovación y desarrollo al interior de la comunidad

- Vincularse en red, con el uso de las herramientas de cualificación e inclusión social a partir de la comunicación digital, esto es: listas de correo, blogs, cursos online y foros de discusión sobre temas relevantes a nivel local, regional e internacional.

- Desarrollar el talento, en este sentido, la conectividad y el acceso a la información promueven la capitalización del conocimiento individual en función del grupo social.

- La promoción de distintas modalidades de enseñanza-aprendizaje de acuerdo a los procesos individuales de aprehensión del conocimiento.

- Brindar un escenario alternativo para el bienestar psicológico y social; los humanos somos una especie que prioriza los vínculos sociales como mecanismo de adaptación exitosa, los KVD acercan a las personas a través de las diferentes plataformas de red social que existen.

- El aprendizaje digital a través de los KVD facilitan el acceso posterior a programas de inclusión social y de salud, como lo es la Telemedicina y las redes sociales temáticas en salud mental y bienestar social que favorecen intereses de la comunidad.

- Los KVD y la inserción social que proporcionan a las comunidades rurales apartadas, facilitan el desarrollo de procesos vinculados al tejido social y el postconflicto a través de estrategias de comunicación social que faciliten educación política en contextos de tolerancia y equidad.

Finalmente uno de los retos que se plantea para los KVD está relacionado al uso adecuado de la conectividad, esto es la responsabilidad ética y social que debe estar presente para el acceso activo a las comunidades virtuales; en este aspecto, es necesario precisar los derechos de autor y la privacidad de las personas, el respeto a las fuentes originales si un recurso en particular se va a compartir en una red social o a través de un blog, imagen o video; el respeto frente al derecho de participar u opinar $y$ expresar percepciones individuales o colectivas en la comunidad digital, debe ser el núcleo central la comunicación virtual. Por tanto, la tolerancia a diferencia y el respeto por el espacio digital del otro como por la apropiación de contenidos debe ser constitutivo en la inclusión digital. Al respecto los KVD deben educar para que los entornos virtuales favorezcan el respeto al otro digital de la misma forma como se respeta al otro real.

\section{REFERENCIAS}

Baym, N. K. (2010) Users of the world, unite! The challenges and opportunities of Social Media. Business Horizons. 53:59-68.

Bercovici, J. (2010) Personal connections in the digital age. Cambridge; Malden, Mass: Polity.

Christakis, N. A., Fowler, J. H. (2016) Lineamientos en TIC. Pacto Social por la Educación. Plan Nacional Decenal de Educación PNDE 2006 2016, Bogotá, Colombia.

Kaplan, A. M. \& Haenlein, M. (2015) Higher-Order Mentalising and Executive Functioning. Personality and individual differences 86:6-14.

Meshi, D; Tamir, D. I \& Heekeren, H. R. (2015) The Emerging Neuroscience of Social Media. Trends in cognitive sciences 19(12):771-82.

Pinker, S. (2009) How the mind works. New York, N.Y.: W.W. Norton.

Reid, E. (1991) Communication and Community on Internet Relay Chat. Electropolis.

Roberts, G. (2015) Human cooperation: the race to give. Current biology: CB 25(10):R425-7.

Small, G \& Vorgan, G. (2008) iBrain: surviving the technological alteration of the modern mind. New York: Collins Living

Walker, S. K. (2011) Connected: the surprising power of our social networks and how they shape our lives. Journal of Family Theory \& Review 3 (3) 220-224- doi: 10.1111/j.17562589.2011.00097.x

Whocoined, J. (2010) 'social media'? Webpioneers compete for credit. Comunicaciones., Ministerio de Tecnologías de la Información y las comunicaciones, Bogotá, Colombia. 